\title{
Ectopic Expression of a Salt-Inducible Gene, LcSAIN3, from Sheepgrass Improves Seed Germination and Seedling Growth under Salt Stress in Arabidopsis
}

\author{
Xiaoxia Li ${ }^{1,+} \mathbb{\oplus}$, Weiguang Yang ${ }^{2,+}{ }^{+}$Junting Jia ${ }^{3}$, Pincang Zhao ${ }^{4} \mathbb{D}$, Dongmei Qi ${ }^{1}$, Shuangyan Chen ${ }^{1} \mathbb{C}$, Li Cheng ${ }^{5}$, \\ Liqin Cheng ${ }^{1, *}$ and Gongshe Liu ${ }^{1}$ \\ 1 Key Laboratory of Plant Resources, Institute of Botany, Chinese Academy of Sciences, Beijing 100093, China; \\ lixx2013@ibcas.ac.cn (X.L.); qidm@ibcas.ac.cn (D.Q.); sychen@ibcas.ac.cn (S.C.); liugs@ibcas.ac.cn (G.L.) \\ 2 College of Animal Science and Veterinary Medicine, Helongjiang Bayi Agricultural University, \\ Daqing 163000, China; anda580@163.com \\ 3 Guangdong Provincial Key Laboratory for Crop Germplasm Resources Preservation and Utilization, \\ Agro-Biological Gene Research Center, Guangdong Academy of Agricultural Sciences, Guangzhou 510640, \\ China; jiajunting123@126.com \\ 4 College of Management Science and Engineering, Hebei University of Economics and Business, \\ Shijiazhuang 050062, China; zhaopincang@163.com \\ 5 Institute of Hulun Buir Forestry and Grassland Science Research, Hunlunbeier 021008, China; \\ cheng1010li@sina.com \\ * Correspondence: lqcheng@ibcas.ac.cn; Tel.: +86-10-6283-6242 \\ check for \\ + These authors have contributed to this work equally.
}

updates

Citation: Li, X.; Yang, W.; Jia, J.; Zhao, P.; Qi, D.; Chen, S.; Cheng, L.; Cheng, L.; Liu, G. Ectopic Expression of a Salt-Inducible Gene, LcSAIN3, from Sheepgrass Improves Seed Germination and Seedling Growth under Salt Stress in Arabidopsis. Genes 2021, 12, 1994

https://doi.org/10.3390/genes12121994

Academic Editors: Zhen Wang, Zhipeng Liu and Wenxian Liu

Received: 28 October 2021

Accepted: 14 December 2021

Published: 16 December 2021

Publisher's Note: MDPI stays neutral with regard to jurisdictional claims in published maps and institutional affiliations.

Copyright: (c) 2021 by the authors. Licensee MDPI, Basel, Switzerland. This article is an open access article distributed under the terms and conditions of the Creative Commons Attribution (CC BY) license (https:// creativecommons.org/licenses/by/ $4.0 /)$.
Abstract: Sheepgrass is a perennial native grass species in China, and it can tolerate high levels of salt stress with an aggressive and vigorous rhizome system. Many salt-stress-responsive genes have been identified in sheepgrass. In this study, we report the cloning and characterization of a novel salt-induced gene, LCSAIN3 (Leymus chinensis salt-induced 3), from sheepgrass. Expression analysis confirmed that $L C S A I N 3$ was induced by PEG, ABA, and salt treatments, and the expression of LCSAIN3 was significantly increased in salt-tolerant germplasms under salt treatment. Subcellular localization analysis indicated that the GFP-LcSAIN3 protein was mainly localized in the chloroplasts. The heterologous expression of LcSAIN3 in Arabidopsis increased the seed germination rate of transgenic plants under salt, ABA, and mannitol treatments. The seedling survival rate, plant height, and fresh weight of the transgenic plants were higher than those of WT plants under salt stress. The overexpression of $L c S A I N 3$ caused a relatively high accumulation of free proline, enhanced SOD activity, and led to the upregulation of several stress-responsive genes such as AtRD26, AtRD29B, AtSOS1, and AtP5CS1. These results suggest that LCSAIN3 could be a potential target for molecular breeding to improve plants' salt tolerance.

Keywords: sheepgrass; chloroplast; salt stress; seed germination

\section{Introduction}

Soil salinization is a severe problem that affects plant growth, development, and productivity worldwide [1]. The effects of salt on plants lead to osmotic, ion toxicity, and oxidative stress [1-3]. Changes in physiological, biochemical, cellular, and molecular processes in plants under salt stress have been investigated by many researchers [2,4-7], while genetic sources for salt tolerance development in crops have also been studied [8]. Extensive numbers of transcription-factor-encoding genes have been identified in response to salt stress, including DREB, bZIP, NAC, and MYB family genes [9-13], and overexpressing these genes can enhance salt stress tolerance in transgenic plants [2,14-17]. Previous studies have suggested that the transcription factors can activate many stress-induced genes, such as LEA genes (RD26, RD29A, RD29B, and $R A B 18)$ and proline biosynthesis genes 
(P5CS), and that LEA proteins are mainly involved in protection to desiccation by acting as cellular dewatering protectants under stress conditions. Proline plays an important role in osmoregulation and can also be used as an active oxygen scavenger to stabilize protein and membrane structures under pressure $[2,18,19]$. Molecular regulatory networks related to salt stress are complex and have not been fully explored [20]; thus, mining key and novel salt-tolerance-related genes is required for developing breeding strategies to enhance salt stress tolerance in crops.

Sheepgrass (Leymus chinensis (Trin.) Tzvel) is a perennial gramineous plant species belonging to the Leymus, Triticeae, and Poaceae classification groups and is widely distributed on the eastern Eurasian steppe [21]. This species can survive when the soil moisture content is less than $6 \%$ in the dry season and grows well in environments of $600 \mathrm{mmol} / \mathrm{L}$ $\mathrm{NaCl}$ and $175 \mathrm{mmol} / \mathrm{L} \mathrm{Na}_{2} \mathrm{CO}_{3}$ [22-24]. Many stress-induced genes from sheepgrass have been identified and characterized using transcriptome sequencing, including $L C D R E B 2$, LcDREB3a, LcDREB21, LcMYB1, LcWRKY5, LcP5CSs, LcbHLH92, and LcSAMDCs [16,25-27]. In addition, several novel genes were discovered in sheepgrass, such as the LcSAIN1 and LcSAIN2 genes, which can improve the greening rate of cotyledons, root elongation, plant height, and survival rates under salt stress in their transgenic plants [28,29]; the ectopic expression of LcFIN1 and LcFIN2 significantly increases freezing stress tolerance in transgenic Arabidopsis and rice [24,30].

Here, we characterize a novel gene, LcSAIN3, from sheepgrass; LcSAIN3 has low homology with LcSAIN1 and LcSAIN2. The expression of LcSAIN3 is induced by salt stress and can improve the salt tolerance of transgenic Arabidopsis. Thus, we propose that LCSAIN3 plays an important role in the salinity stress regulatory network.

\section{Materials and Methods}

\subsection{Plant Materials, Growth Conditions, and Stress Treatment}

Sheepgrass variety Zhongke No. 1 and sheepgrass germplasms with different salt tolerances (salt-tolerant germplasm G53, G25, and G16 and salt-sensitive germplasm G13 and G87) were cultivated by the Institute of Botany, the Chinese Academy of Sciences. Sheepgrass, Arabidopsis thaliana (ecotype Columbia (Col-0)), and tobacco (Nicotiana benthamiana) were grown in a soil mixture of peat moss and vermiculite $(2: 1, v / v)$ in a glasshouse, and the plants' growth conditions were followed as previously described [23,28,29]. For the analysis of specific expression in different tissues, the roots, stems, leaves, and seeds were collected from two-year-old sheepgrass plants under normal conditions. For salt, ABA, and dehydration treatments, $400 \mathrm{mM} \mathrm{NaCl}, 100 \mathrm{mM}$ abscisic acid (ABA), and 20\% PEG6000 were applied to 4 -week-old sheepgrass seedlings as previously described $[23,28,29]$. A total of 40 plants for each sample were harvested at $0,1,3,5,12$, and $24 \mathrm{~h}$ after treatments, and three independent sets of samples were collected for each time point. Samples were immediately frozen in liquid nitrogen and stored at $-80^{\circ} \mathrm{C}$.

\subsection{Cloning and Sequence Analysis of the LcSAIN3 Gene}

LcSAIN3 gene (GenBank ID: MN901606) was isolated from sheepgrass and encoded an unknown functional gene. Total RNA was extracted from 4-week-old sheepgrass seedlings under $400 \mathrm{mM} \mathrm{NaCl}$ for $12 \mathrm{~h}$ using a TRIzol kit (TaKaRa, Dalian, China) based on the protocol instructions of the manufacturer, and first-strand cDNA synthesis was performed with a SMART RACE cDNA Amplification Kit (Clontech, Palo Alto, CA, USA) according to the manufacturers' instructions. Full-length LcSAIN3 cDNA was amplified using the primers $5^{\prime}$-GTAGCCCGTGAGGAAGTT- $3^{\prime}$ and 5'-CACTAGAAGGGCCCCGAA-3' ${ }^{\prime}$, with the cDNA of $5^{\prime}$ RACE used as a template. The amplification was carried out following a previously described method $[23,28,29]$, and all of the PCR products were cloned into a pMD19-T vector and sequenced at Sangon Biotech (Shanghai Co., Ltd., Shanghai, China).

The LcSAIN3 sequence was analyzed using the BLAST program of the National Center for Biotechnology Information (NCBI) (https: / / www.ncbi.nlm.nih.gov /, accessed on 3 January 2020), and subcellular localization was predicted using the Plant-mPLoc program [31]. 


\section{3. qRT-PCR Analysis}

Total RNA from Arabidopsis and sheepgrass seedlings was isolated using a TRIzol kit (TaKaRa, Dalian, China) according to the instructions of the manufacturer. Total RNA was reverse-transcribed using a PrimeScript ${ }^{\mathrm{TM}}$ PCR Kit, and the SYBR ${ }^{\circledR}$ PrimeScript TM PCR Kit was used for qPCR following the manufacturer's instructions (TaKaRa, Dalian, China). qPCR was performed using the Roche Light Cycler 480 II (Basel, Switzerland), and the qPCR program was set according to our previously described reports $[23,28,29]$; the data were quantified using the comparative $2^{-\Delta \Delta C T}$ method, as described previously [32]. LcActin and AtActin2 were used as internal controls for assessing the expression levels in sheepgrass and Arabidopsis, respectively. The primers of LCSAIN3 for qRT-PCR were 5'-ACTGGTGTTGGATGATGAGCG-3' and 5'-CGGGAGGAAAGATAGAGGTCG-3' ${ }^{\prime}$. The primers' information for salt-induced genes in Arabidopsis was followed, as previously described $[28,29]$.

\subsection{Subcellular Localization of LcSAIN3}

The open reading frame (ORF) of LcSAIN3 was inserted into the expression vector pMDC45 (containing GFP) using Gateway cloning while the construct was transferred to Agrobacterium tumefaciens EHA105. The Agrobacterium tumefaciens containing the pMDC45-LcSAIN3 (35S::GFP-LcSAIN3) recombinant vector and the pMDC45 empty vector were injected into 4-week-old wild-type tobacco (N. benthamiana) leaves, as described by Gao et al. [24]. GFP fluorescence in tobacco leaves was observed and imaged for 2 to $3 \mathrm{~d}$ after infiltration using an argon laser at $488 \mathrm{~nm}$ (GFP) and 382 bright-field images by confocal microscope Leica TCS SP5 microscope (Leica Microsystems, Wetzlar, Germany) [30].

\subsection{Construct Creation and Plant Genetic Transformation}

The ORF of LcSAIN3 was cloned into a pSN1301 vector, and the construct pSN1301LcSAIN3 was introduced into the Agrobacterium EHA105. Then, the Agrobacterium EHA105 containing the recombinant plasmid was transformed into Arabidopsis following a previously described method [33]. The candidate transgenic Arabidopsis seeds were firstly screened on Murashige and Skoog (MS) solid medium agar supplemented with $30 \mu \mathrm{g} \mathrm{mL}-1$ hygromycin and then further confirmed by PCR analysis using the gene-specific primers.

\subsection{Phenotypic Analysis of Transgenic Plants}

The T3 generation LcSAIN3 transgenic Arabidopsis lines (line 5, line 6, and line 8) were used for further analysis. The seeds were incubated at $4{ }^{\circ} \mathrm{C}$ for $2 \mathrm{~d}$ to break dormancy and then germinated on MS medium supplemented with different concentrations of ABA ( 1 and $2 \mu \mathrm{M})$, mannitol (200 and $300 \mathrm{mM}$ ), and $\mathrm{NaCl}(100,125,150,175$ and $200 \mathrm{mM})$, respectively. The germination rate was calculated daily for $7 \mathrm{~d}$ by observing radical protrusion, and at least 120 seeds from each transgenic line were evaluated. To test the salt tolerance of transgenic Arabidopsis, 3-week-old plants were treated with $200 \mathrm{mM} \mathrm{NaCl}$ for 3 weeks at 3-day intervals, as described previously [34]. Three-week-old transgenic and WT seedlings grown in the MS liquid medium were treated with $150 \mathrm{mM} \mathrm{NaCl}$ after $1 \mathrm{~d}$ and were sampled and used for qPCR analysis. Additionally, AtActin was used as a reference gene.

\subsection{Measurement of Proline Content and (Superoxide Dismutase) SOD Activity}

Proline was measured as previously described [35], and total superoxide dismutase (SOD) activity was measured using nitro blue tetrazolium (NBT) reduction, as described previously $[30,36]$.

\subsection{Statistical Analysis}

The data concerning Arabidopsis seed germination rates and seedling growth parameters, proline content, and SOD activity were subjected to one-way ANOVA using the SPSS 21.0 program (IBM, Chicago, IL, USA). 


\section{Results}

\subsection{Isolation and Sequence Analysis of LcSAIN3}

Our previous studies identified many stress-induced genes from sheepgrass using transcriptome sequencing techniques [23]. In this study, the full-length cDNA was obtained from sheepgrass by RACE. The gene (GenBank ID: MN901606) is $847 \mathrm{bp}$ long and encodes 198 amino acids, and BLAST analysis shows it has high homology $(72 \%)$ with a wheat cDNA clone, WT004_K04 (GenBank ID: AK331493). The amino acid sequence shows 52\% homology with the predicted protein product of Triticum turgidum subsp. Durum (GenBank ID: VAH97959.1) and 40.52\% with hypothetical protein CFC21_054876 of Triticum aestivum (GenBank ID: KAF7060855.1) using a BLASTX search (Figure 1). Furthermore, it shared low homology to LcSAIN1 (9.67\%) and LcSAIN2 (21.93\%) (Figure S1). These results imply that it is a novel protein with unknown function, and it was named LcSAIN3 (L. chinensis salt-induced 3).

\section{$\stackrel{0.05}{.}$}

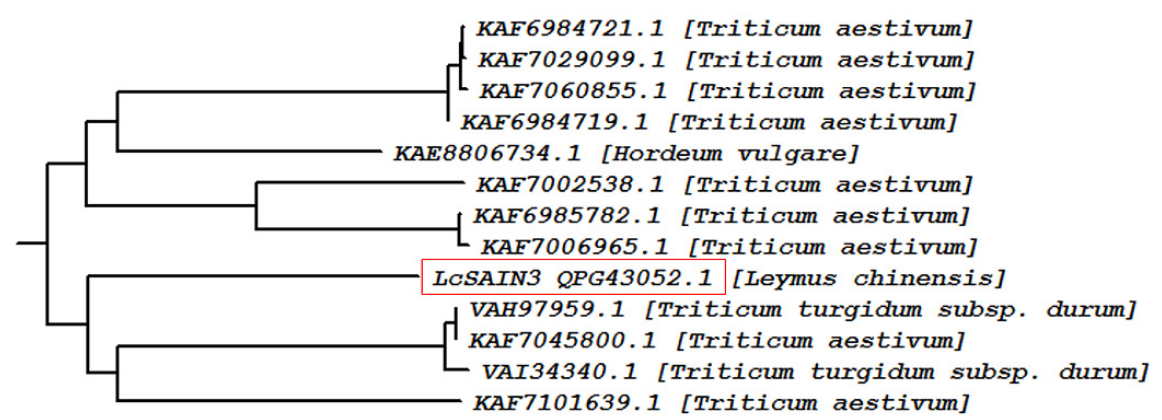

Figure 1. Phylogenetic analysis of LcSAIN3 and homologs was constructed based on amino acid sequences using the neighbor-joining method.

\subsection{Expression Analysis of LcSAIN3}

The qRT-PCR analysis showed that LCSAIN3 was highly expressed in the stems of two-year-old sheepgrass plants under normal conditions (Figure 2a). To determine whether LCSAIN3 was responsive to stress in sheepgrass, 4-week-old sheepgrass seedlings were exposed to salt, $\mathrm{ABA}$, and dehydration stresses and were sampled at different time intervals. QRT-PCR results showed that the expression level of LCSAIN3 was significantly upregulated at $3 \mathrm{~h}$ and reached the highest level after $5 \mathrm{~h}$ of $\mathrm{NaCl}$ treatment, approximately 5.4-fold (Figure $2 b$ ). It is different from the quick response of salt stress treatments; the expression of LcSAIN3 slowly increased up to 15.5 -fold at $12 \mathrm{~h}$ and 2.2 -fold at $5 \mathrm{~h}$ of treatment with ABA and PEG, respectively (Figure 2c,d).

\subsection{Subcellular Localization of LcSAIN3}

To determine the subcellular localization of LcSAIN3, the protein was predicted by the Plant-mPLoc program (http:/ / www.csbio.sjtu.edu.cn/bioinf/plant-multi/, accessed on 3 January 2020); it demonstrated that LcSAIN3 was a chloroplast protein. To determine the actual subcellular localization of LcSAIN3 in vivo, the ORF sequence was inserted into a pMDC45 vector fused to a GFP reporter gene under the control of the CaMV 355 promoter, and the construct of 35S::GFP-LcSAIN3 was infiltrated into tobacco (Nicotiana tabacum) leave cells (using 35S-GFP as the control). As shown in Figure 3, the green fluorescent signals from the 35S::GFP-LCSAIN3 fusion protein and autofluorescent signals of chloroplasts were merged together; these results demonstrate that LcSAIN3 is a chloroplast-localized protein. 
a

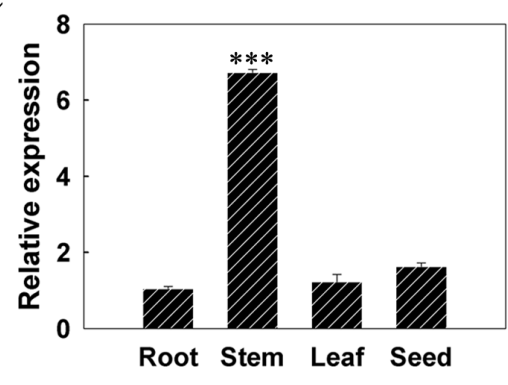

c

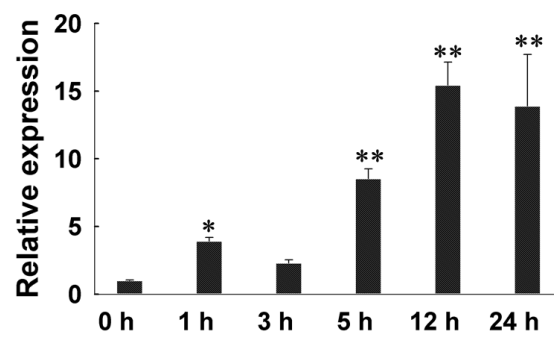

b
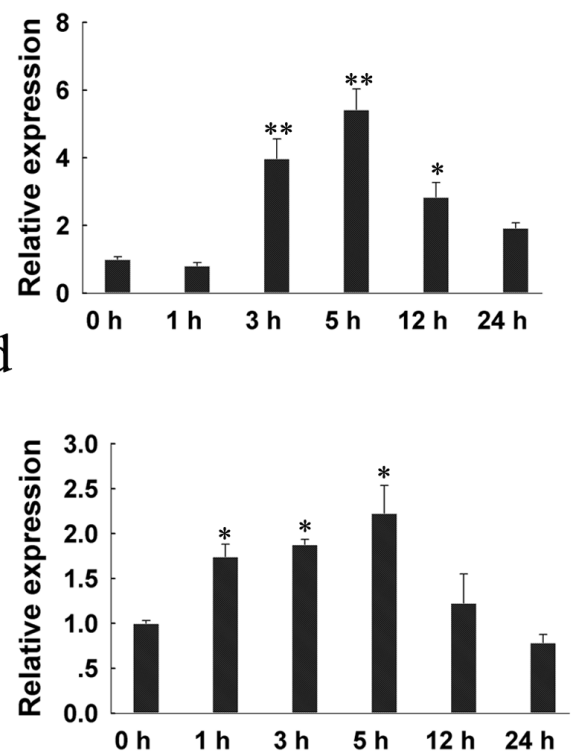

Figure 2. Expression patterns of $L c S A I N 3$ in sheepgrass tissues and abiotic stress. (a) Expression of LcSAIN3 in roots, stems, leaves, and seeds. (b-d) Expression patterns of the LcSAIN3 gene under salt, ABA, and PEG treatments. The sheepgrass Actin gene is used as the internal reference gene for normalization, and data represent means \pm SDs of three independent biological replicates. ${ }^{* * *},{ }^{* *}$, and ${ }^{*}$ indicate significant differences at $p<0.001, p<0.01$, and $p<0.05$, respectively.

GFP
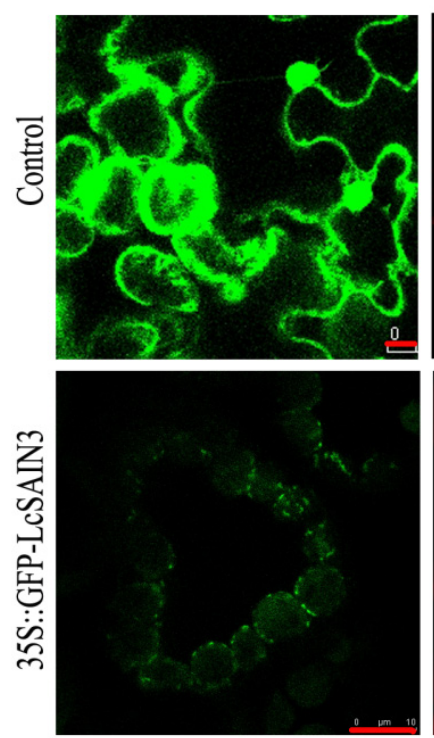

Chloroplast
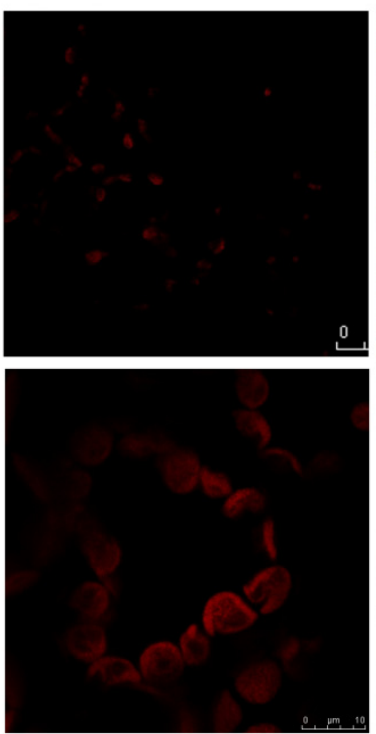

Merged
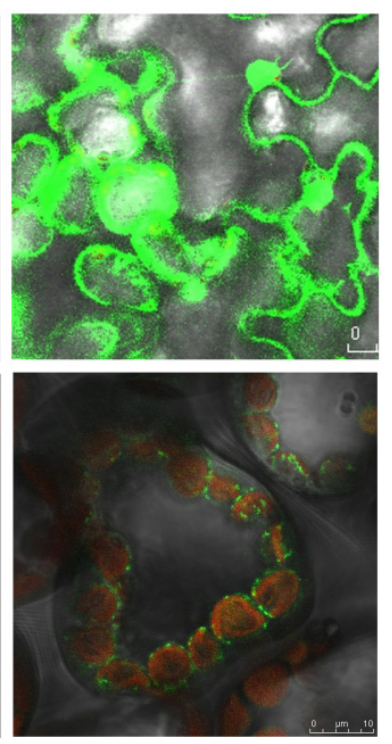

Figure 3. The subcellular localization of LcSAIN3. The GFP signals arising from the GFP control (upper) and 35S::GFP-LcSAIN3 fusion protein (bottom lane) expressed in tobacco leave cells were detected under fluorescent-field illumination, chloroplast autofluorescence, and an overlay using a confocal microscope. Red signals represent chloroplast autofluorescence. Scale bar $=10 \mu \mathrm{M}$.

\subsection{Overexpression of LcSAIN3 in Arabidopsis Improves Seed Germination under Salt Stress}

To further understand the function of $L c S A I N 3$ in response to salt stress, the Arabidopsis LcSAIN3-overexpressed lines were generated. Three T3 transgenic lines (L5, L6, and L8) with higher LcSAIN3 transcript levels by qRT-PCR were selected for further investigation (Figure S2). To evaluate the performance of LcSAIN3 transgenic plants in response to 
salt stress, the seeds of WT and LcSAIN3-overexpressed plants were germinated on MS media supplemented with different concentrations of $\mathrm{NaCl}(0,100,125,150,175$, and $200 \mathrm{mM}$ ) after $2 \mathrm{~d}$ of stratification. As shown in Figure 4, no obvious differences were detected between WT and transgenic plants on MS medium without $\mathrm{NaCl}$. However, the germination rates of the LCSAIN3-overexpressing lines were significantly higher than those of WT plants in the presence of $\mathrm{NaCl}$ (Figure 4a). In addition, three transgenic lines (L5, L6, and L8) showed significantly higher germination rates $(92 \%, 86 \%$, and $81 \%$, respectively) than WT plants under $150 \mathrm{mM} \mathrm{NaCl}(\sim 55 \%)$ (Figure $4 \mathrm{~b}$ ). Thus, LcSAIN3 overexpression in Arabidopsis reduces sensitivity to salt stress at the seed germination stage.

a
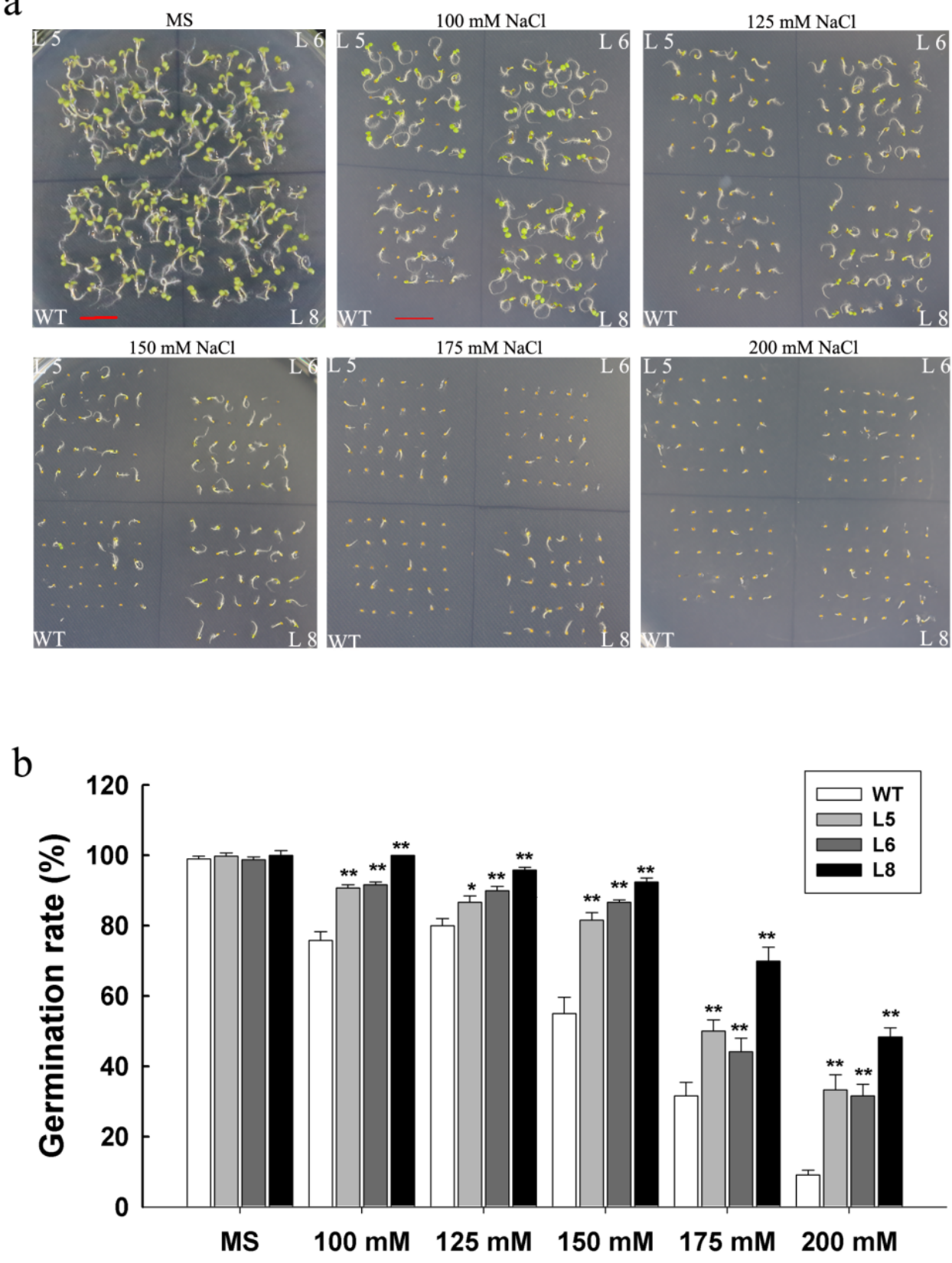

Figure 4. Salt stress tolerance of LcSAIN3 transgenic Arabidopsis plants at germination stage. (a) Phenotypes of WT (wild-type) and LcSAIN3-overexpressing lines (L5, L6, and L8 seeds) in MS medium with $0,100,125,150175$, or $200 \mathrm{mM} \mathrm{NaCl}$. (b) Seed germination rate under salt stress. Thirty seeds were allowed to grow for seven days after sowing, and each column represents an average of three replicates, and the bars indicate standard deviations (SDs). ${ }^{* *}$ and ${ }^{*}$ indicates significant differences in comparison with the control at $p<0.01$ and $p<0.05$. Scale bar $=1 \mathrm{~cm}$. 


\subsection{LcSAIN3 Overexpression in Arabidopsis Enhanced Seed Germination under ABA and Osmotic Stress}

Since the expression of the LCSAIN3 gene was significantly induced by ABA and osmotic stress (Figure 2), the tolerance to $\mathrm{ABA}$ and mannitol of transgenic lines was examined. Likewise, when the Arabidopsis seeds were subjected to $2 \mu \mathrm{M}$ ABA or 200 and $300 \mathrm{mM}$ mannitol, the germination rate of WT seeds was only $57 \%$, while the germination rates of the three LCSAIN3-overexpression lines, i.e., L5, L6, and L8 seeds, were 79\%, 87\% and $75 \%$ under ABA treatment, respectively $(p<0.05)$ (Figure $5 \mathrm{a}, \mathrm{c})$. With treatments of 200 and $300 \mathrm{mM}$ mannitol, the germination of WT plants was significantly lower than the transgenic plants $(p<0.05)$ (Figure $5 \mathrm{~b}, \mathrm{~d})$. This implies that over-expression of LcSAIN3 reduces the sensitivity to $\mathrm{ABA}$ and osmotic stresses at the seed germination stage.

a

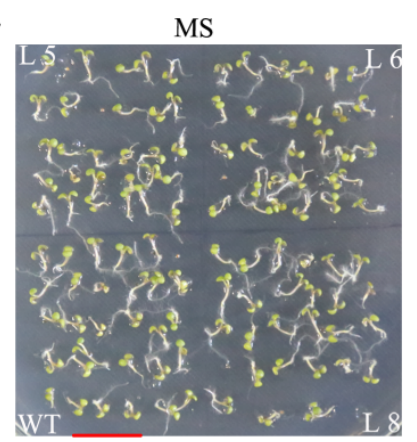

$\mathrm{b}$

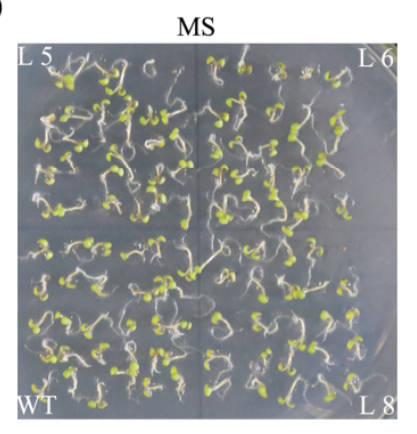

C

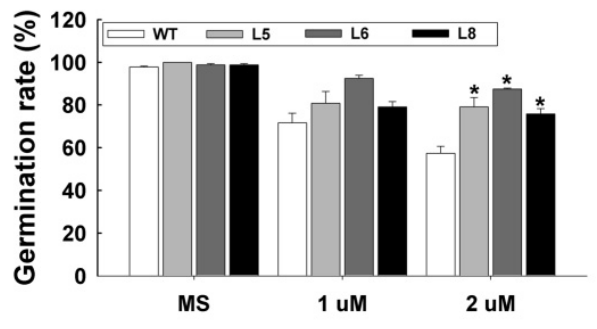

$1 \mu \mathrm{M} \mathrm{ABA}$

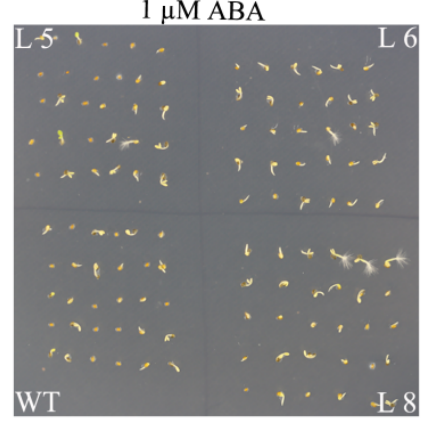

200 mM Mannitol

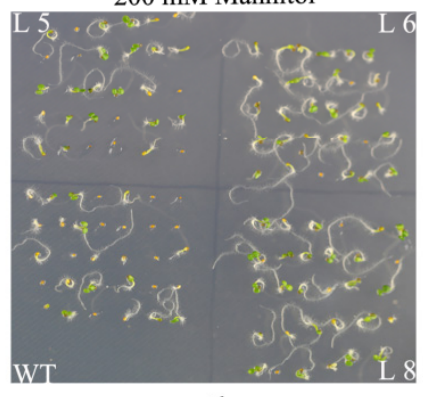

$2 \mu \mathrm{M}$ ABA
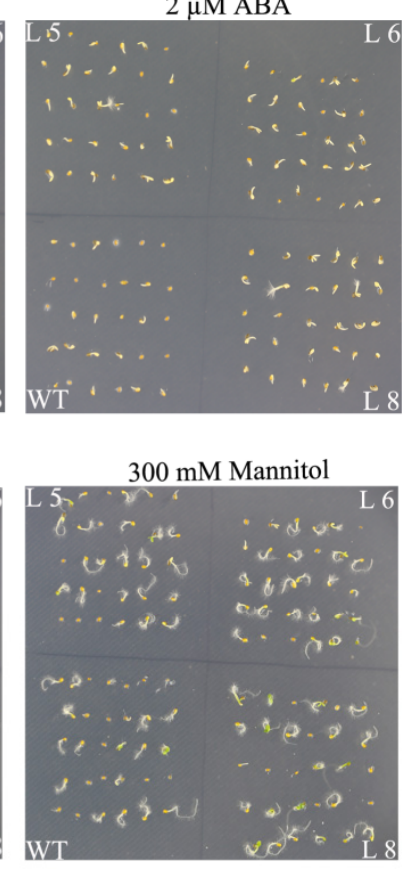

d

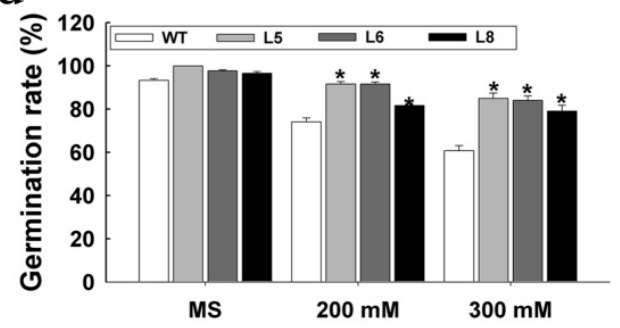

Figure 5. ABA and mannitol stresses tolerance of LCSAIN3 transgenic Arabidopsis plants at germination stage. $(\mathbf{a}, \mathbf{c})$ seed germination rates of the WT and $L c S A I N 3$-expressing lines were measured on MS media and MS media containing 1 and $2 \mu \mathrm{M}$ ABA. $(\mathbf{b}, \mathbf{d})$ Seed germination rates of the WT and LcSAIN3-expressing lines were measured on MS media and MS media containing 200 and 300 $\mathrm{mM}$ mannitol. Thirty seeds were allowed to grow for seven days after sowing, and each column represents an average of three replicates; the bars indicate standard deviations (SDs). * indicates significant differences in comparison with the control at $p<0.05$. Scale bar $=1 \mathrm{~cm}$.

\subsection{LcSAIN3 Overexpression Enhances Tolerance to Salt Stress in Arabidopsis Seedlings}

To investigate the performance of LcSAIN3 transgenic lines in response to salt stress at the seedling stage, 3-week-old Arabidopsis plants were treated with $200 \mathrm{mM} \mathrm{NaCl}$ for 3 weeks at 3-day intervals [34]. After exposure to $\mathrm{NaCl}$ treatment, the growth of the transgenic lines and WT were all inhibited, but the sensitivity of the LcSAIN3-overexpression 
lines to salt stress was reduced. For example, most of the WT seedlings were bleached and wilted after the 3-week salt treatment; in contrast, most seedlings of three LcSAIN3overexpression lines (L5, L6, and L8) survived and had both green and yellow leaves. The three transgenic lines had significantly higher survival rates $(93 \%, 87 \%$, and $98 \%$ ) compared to that of WT plants $(\sim 30 \%)$ (Figure $6 a, b)$. Furthermore, plant height and fresh weight were significantly greater for the transgenic plants than WT plants (Figure 6c,d). These data suggest that overexpression of LCSAIN3 helped to enhance salt tolerance in transgenic Arabidopsis.

a
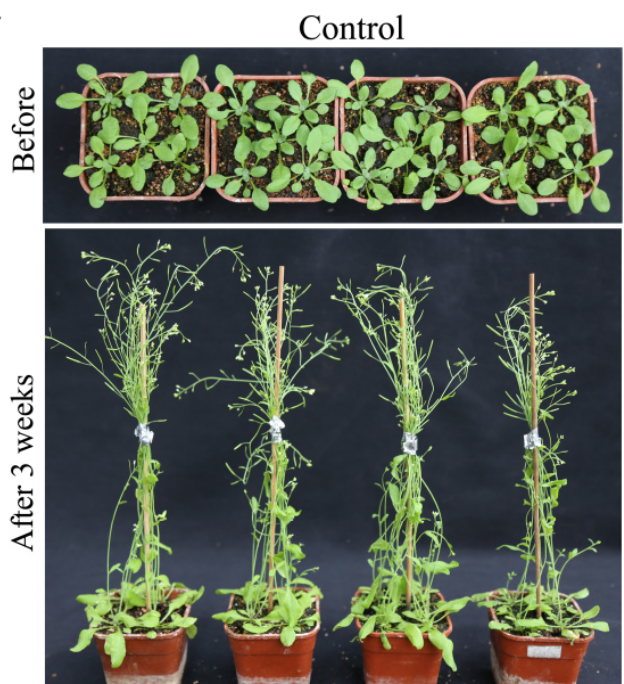

WT
L5
L6

L8

b
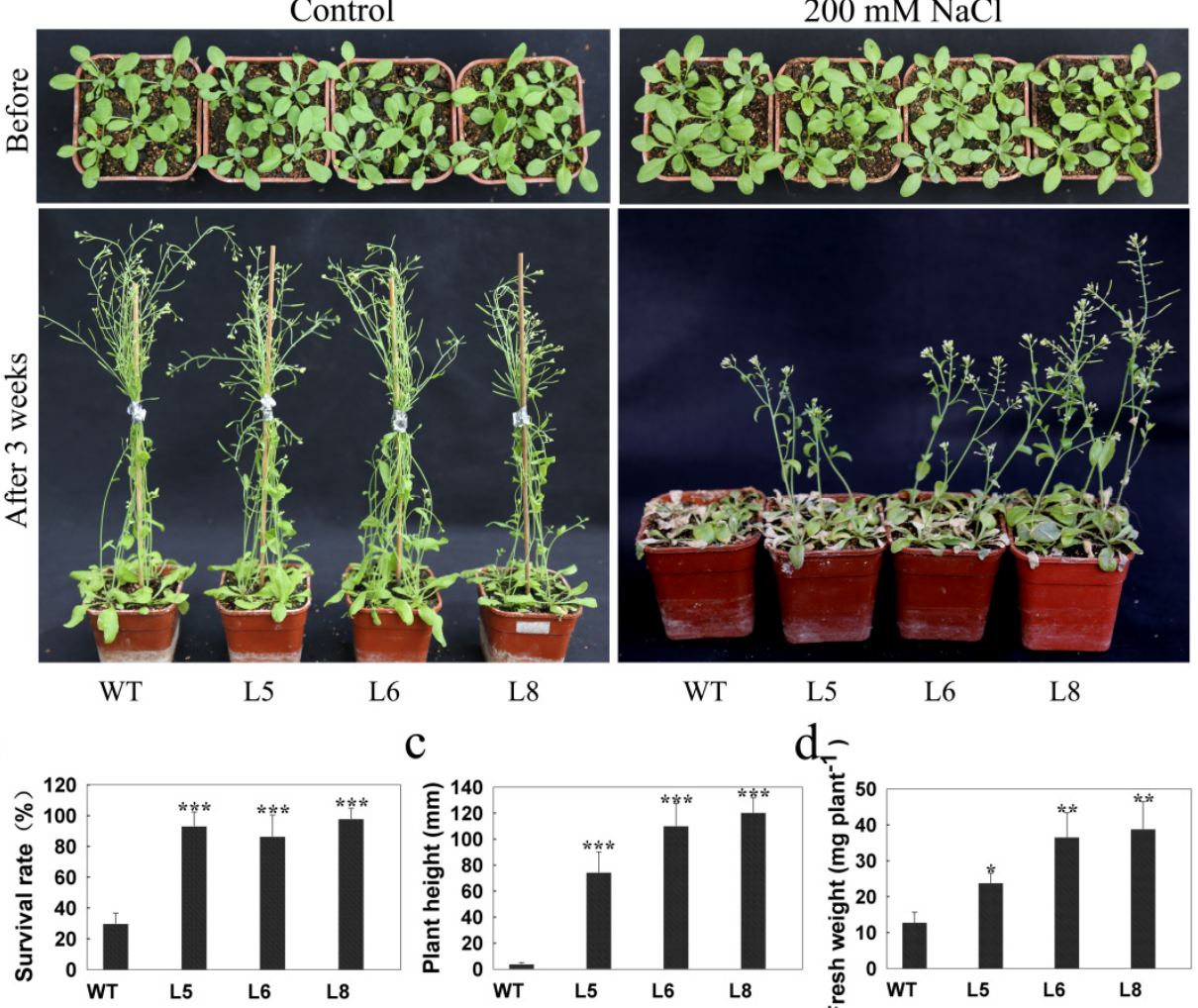

WT

L5

L6

L8

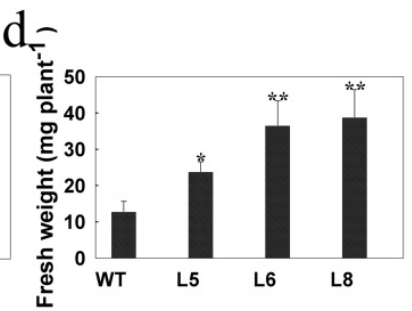

Figure 6. Enhanced tolerances to salt stress in LCSAIN3-overexpressing Arabidopsis at the seedling stage. (a) Three-week-old seedlings in soil were treated with or without $200 \mathrm{mM} \mathrm{NaCl}$ for 3 weeks. The seedling survival rates (b) and plant height (c) and plant weight (d) were scored after the 3-week treatment. The mean and standard error were obtained from three biological replicates, ${ }^{* * *}, * *$, and ${ }^{*}$ indicate significant differences at $p<0.001, p<0.01$, and $p<0.05$, respectively..

\subsection{LcSAIN3 Regulates Proline Accumulation and SOD Activity in Response to Salt Stress}

To further characterize the possible mechanism that may be responsible for improving tolerance of the transgenic plants to salt stress, we assayed proline content and SOD (a major antioxidant enzyme) activity in 3-week-old transgenic and WT seedlings treated with $200 \mathrm{mM} \mathrm{NaCl}$ for $2 \mathrm{~d}$. Under the treatment with $\mathrm{NaCl}$, the proline content in both transgenic plants and WT plants increased, but the transgenic plants accumulated a higher level of proline than WT plants, especially in L5 and L6 (Figure 7a). Further, the SOD activity was significantly higher in the transgenic L5 and L6 plants than in the WT plants under salt stress $(p<0.01)$ (Figure $7 \mathrm{~b})$. Taken together, our results indicate that LcSAIN3 overexpression in Arabidopsis could promote proline content and SOD activity under salt stress. 
$\mathrm{a}$

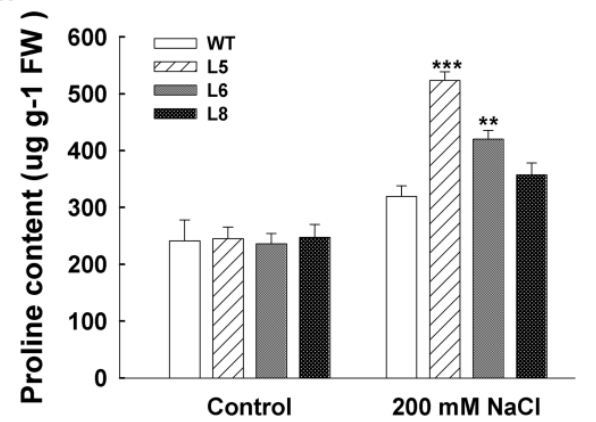

b

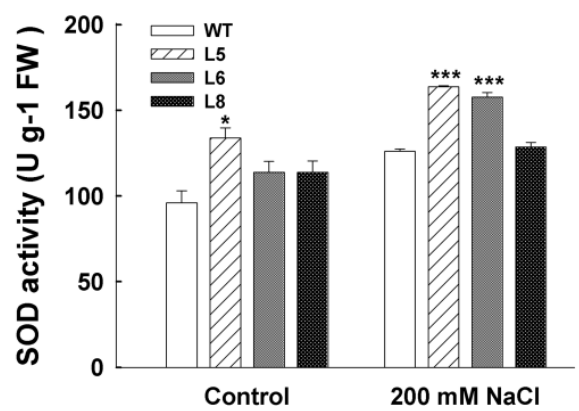

Figure 7. Physiological index analyses of LcSAIN3-overexpressing plants. Proline contents (a) and antioxidant enzymes levels (b) in transgenic and WT seedlings exposed to $200 \mathrm{mM} \mathrm{NaCl}$ for $2 \mathrm{~d}$. The bars indicate standard deviations, and the results are from three independent biological replicates. $* * * * *$, and * indicate significant differences in comparison with the control at $p<0.001, p<0.01$, and $p<0.05$, respectively.

\subsection{LcSAIN3 Overexpression Alters the Expression of Salt-Responsive Genes in Arabidopsis Plants}

To clarify the effect of LcSAIN3 on the molecular basis of salinity stress response, the expression levels of several known salt-stress-responsive marker genes were compared between the transgenic Arabidopsis (averaged OE lines, average expression levels of transgenic line 5,6,8) and WT plants using qRT-PCR under salt stress. As shown in Figure 8, the transcripts of $R D 26$ and $R D 29 B$ genes increased in the transgenic plants compared with WT plants under salt stress. Similarly, the expression levels of SOS1 also significantly increased in the transgenic lines (Figure 8). Taken together, these results suggest that LcSAIN3 may improve the salt stress tolerance of transgenic plants by upregulating the expression of salt-stress-responsive genes.

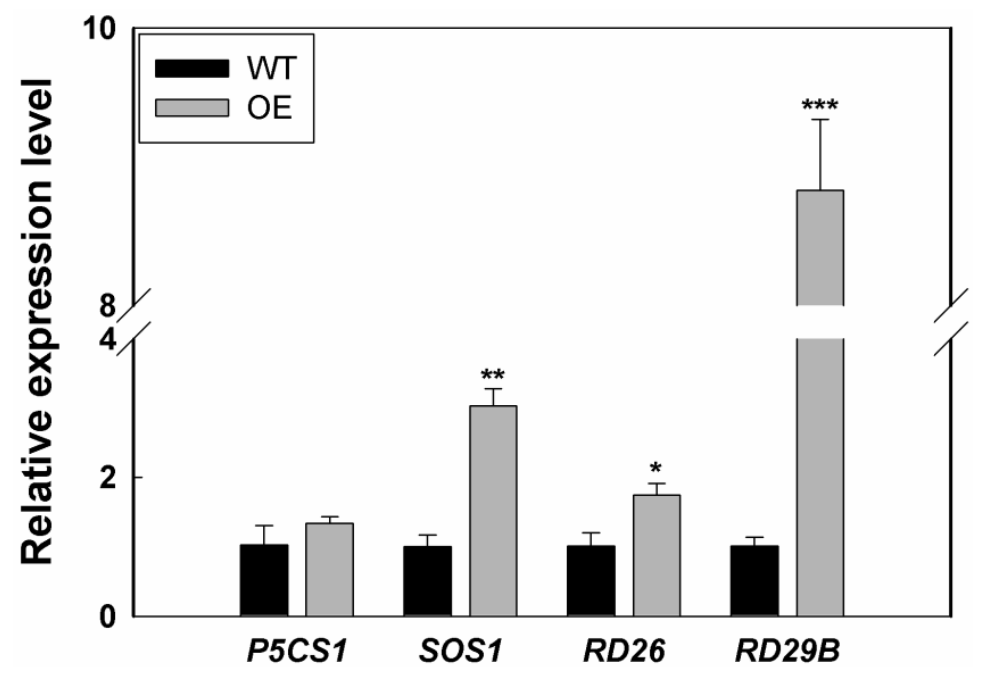

Figure 8. Relative expression levels of five stress-associated genes in the LcSAIN3-overexpressing plants compared with wild-type under salt stress. Real-time PCR analysis of six salt-stress-induced genes in transgenic Arabidopsis plants in the presence of $150 \mathrm{mM} \mathrm{NaCl}$. AtActin2 was used as the reference gene. ${ }^{* * *}, * *$, and ${ }^{*}$ indicate significant differences in comparison with the control at $p<0.001$, $p<0.01$, and $p<0.05$, respectively.

\subsection{The Expression of LcSAIN3 in Sheepgrass Germplasms with Different Salt Tolerance}

To investigate the expression level of LcSAIN3 in sheepgrass germplasms with different salt tolerance, we performed qRT-PCR analysis. The results revealed that the relative expression levels of LcSAIN3 were significantly induced in salt tolerant germplasms (G53, 
G25, and G16) after $4 \mathrm{~h}$ of salt treatment, while the relative expression levels in salt sensitive germplasms (G87, G13) were changed slightly under salt treatment (Figure 9). These results indicate that LcSAIN3 may play important role in response to salt stress in sheepgrass germplasms.

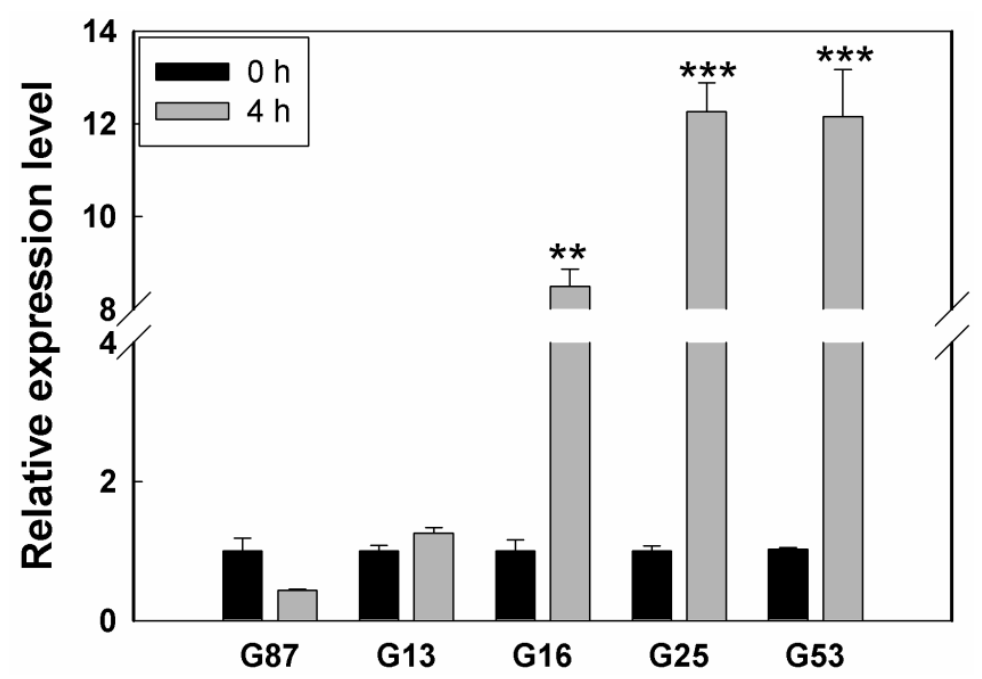

Figure 9. Expression analysis of LCSAIN3 in sheepgrass germplasms with different salt tolerance. G53, G25 and G16 are salt tolerant germplasms, and G13 and G87 are salt sensitive germplasms. ${ }^{* * *}$, and ${ }^{* *}$ indicate significant differences in comparison with the control at $p<0.001$, and $p<0.01$, respectively.

\section{Discussion}

Sheepgrass is an important forage grass as well as an environmentally friendly native grass species in China. It has a high yield with high protein content, better palatability, strong regeneration ability, strong cold and drought resistance, as well as salt-alkali resistance [21,23]. Our previous studies have demonstrated that the novel genes LcFIN1 and LcFIN2 from sheepgrass enhance tolerance to low temperature in Arabidopsis and rice, while overexpressing LcSAIN1 and LcSAIN2 could enhance the salt stress resistance of transgenic plants compared with wild-type plants [24,28-30]. The isolated salt-induced gene, LcSAIN3, from sheepgrass in the present study has high homology (72\%) only with a wheat cDNA clone, WT004_K04 (GenBank ID: AK331493), and the amino acid sequence of LcSAIN3 shows 52\% homology with a predicted protein product of T. turgidum subsp. Durum (Figure 1). In our previous study, two salt-stress-induced LcSAIN1 and LcSAIN2 proteins were mainly localized in the cell nucleus [28,29], while the LcSAIN3 gene encoded a chloroplast-targeted protein (Figure 3). Further, LcSAIN3 had low homology with LcSAIN1 and LcSAIN2 proteins (Figure S1), and the transcript abundance of the LcSAIN3 gene was significantly induced by salinity, PEG, and ABA treatments (Figure 2).

The LcSAIN3 gene is overexpressed in Arabidopsis as genetic transformation in sheepgrass is still very difficult [37]. Our results showed that overexpression of LcSAIN3 in Arabidopsis led to an increase in tolerance to $\mathrm{NaCl}$, ABA and PEG treatments, as reflected by the increase in the germination rate (Figures 4 and 5). Further, the survival rates, fresh weight, and plant length of transgenic plants under salt stress were markedly increased during the seedling growth stage (Figure 6). Moreover, LcSAIN3 expression levels were significantly up-regulated in salt tolerant sheepgrass germplasms under salt stress (Figure 9). Thus, our results suggest that LcSAIN3 may play important role in response to salt stress. Our preliminary results showed that LcSAIN3 is a chloroplast-localized protein (Figure 3). Overexpression of a novel chloroplast protein CEST enhanced the tolerance of transgenic Arabidopsis to multiple environmental stresses [38]; a chloroplast outer envelope protein from Suaeda salsa is also involved in oxidative stress tolerance [39]. In addition, overexpression of chloroplast-localized rice OsRH58 also improved seed 
germination and seedling growth under salt stress conditions by increasing the translation of chloroplast mRNAs [40]. In this study, a novel chloroplast protein, LCSAIN3, plays a positive role in the response to salt, ABA, and PEG stresses and could be used as a gene resource to enhance stress tolerance in wheat and other crops.

The accumulation of proline in plants under salt stress has multiple protective functions, including osmotic protection and ROS scavenging [41]. SOD is proven to be a kind of antioxidant enzyme that plays an important role in scavenging ROS and protects against oxidative stress under salt conditions [20]. Under salt stress conditions, proline levels and SOD activities are higher in the LcSAIN3-overexpression lines than in the wild type (Figure 7), suggesting that LcSAIN3 may be involved in salt stress responses by the accumulation of more proline and higher SOD activity. Other studies have shown that the SOS pathway is a key regulator of $\mathrm{Na}^{+}$homeostasis, for example, via SOS1 [3]. P5CS1 is a key enzyme in the proline biosynthesis pathway, and it functions as a positive regulator in proline accumulation and plant responses to salt tolerance [13,42]. In the present study, the AtSOS1 gene is expressed at much higher levels in the LcSAIN3 transgenic plants than in the WT plants under salt stress and the expression level of AtP5CS1 was induced lightly (Figure 8). Furthermore, in transgenic Arabidopsis plants overexpressing LcSAIN3, the expression of the ABA-dependent genes AtRD26 and AtRD29B are also significantly increased than that in the WT plants under salt stress. These genes have been found to be induced by salinity, and they play important roles in the response to abiotic stress. AtRD29B is involved in ABA-dependent signaling pathways $[43,44]$. These findings indicate that the improved tolerance of the transgenic plants to salinity stress might partly result from the enhanced proline content and SOD activity and the increased expression of salt stress-responsive marker genes (AtSOS1, AtRD26 and AtRD29B etc.).

\section{Conclusions}

In summary, we have characterized a novel chloroplast-localized protein, LcSAIN3; the protein plays a positive role in the salt stress pathway. Overexpression of LcSAIN3 in Arabidopsis elevates seed germination and seedling survival rates under salt stress. The changes of part physiological indices (proline content and SOD activity) and saltresponsive genes (AtSOS1, AtRD29B etc.) of $L c S A I N 3$ overexpression plants may give rise to the improving salt tolerance of transgenic plants.

Supplementary Materials: The following are available online at https:/ / www.mdpi.com/article/ $10.3390 /$ genes $12121994 / \mathrm{s} 1$. All data generated or analyzed during this study are included in this published article and its supplementary information files, namely, Figure S1: Comparison of the amino acid sequences of LcSAIN1, LcSAIN2, and LcSAIN3 proteins and Figure S2: QRT-PCR identification of transgenic Arabidopsis and WT with the LcSAIN3 gene.

Author Contributions: L.C. (Liqin Cheng) and G.L. planned and designed the research. X.L. and W.Y. performed the experiments. J.J., P.Z. and D.Q. made contributions to plant material collection and experimental management. L.C. (Li Cheng) and S.C. analyzed the data. X.L. and W.Y. wrote the manuscript. L.C. (Liqin Cheng) and G.L. edited the manuscript and gave the final approval to the manuscript. All authors have read and agreed to the published version of the manuscript.

Funding: This work was supported by the National Natural Science Foundation of China (No. 32071869), the Key Projects in Science and Technology of the Inner Mongolia (2021ZD0031), and the Strategic Priority Research Program of the Chinese Academy of Science (Grant No. XDA26030202).

Institutional Review Board Statement: Not applicable.

Informed Consent Statement: Not applicable.

Data Availability Statement: The gene sequence is available in the NCBI gene database under accession number MN901606 (https:/ /www.ncbi.nlm.nih.gov/nuccore/MN901606, accessed on 3 January 2020).

Conflicts of Interest: The authors declare that there are no conflict of interest. 


\section{References}

1. Munns, R.; Tester, M. Mechanisms of salinity tolerance. Annu. Rev. Plant Biol. 2008, 59, 651-681. [CrossRef] [PubMed]

2. Deinlein, U.; Stephan, A.B.; Horie, T.; Luo, W.; Xu, G.; Schroeder, J.I. Plant salt-tolerance mechanisms. Trends Plant Sci. 2014, 19, 371-379. [CrossRef] [PubMed]

3. Isayenkov, S.V.; Maathuis, F.J.M. Plant Salinity Stress: Many Unanswered Questions Remain. Front Plant Sci. 2019, 10, 80. [CrossRef] [PubMed]

4. Xiong, L.; Schumaker, K.S.; Zhu, J.K. Cell signaling during cold, drought, and salt stress. Plant Cell 2002, 14 (Suppl. 1), S165-S183. [CrossRef]

5. Reddy, A.S.; Ali, G.S.; Celesnik, H.; Day, I.S. Coping with stresses: Roles of calcium- and calcium/calmodulin-regulated gene expression. Plant Cell 2011, 23, 2010-2032. [CrossRef] [PubMed]

6. Zhao, C.; Zayed, O.; Zeng, F.; Liu, C.; Zhang, L.; Zhu, P.; Hsu, C.C.; Tuncil, Y.E.; Tao, W.A.; Carpita, N.C.; et al. Arabinose biosynthesis is critical for salt stress tolerance in Arabidopsis. New Phytol. 2019, 224, 274-290. [CrossRef] [PubMed]

7. Ma, X.; Liang, X.; Lv, S.; Guan, T.; Jiang, T.; Cheng, Y. Histone deacetylase gene PtHDT902 modifies adventitious root formation and negatively regulates salt stress tolerance in poplar. Plant Sci. 2020, 290, 110301. [CrossRef]

8. Isayenkov, S.V. Genetic sources for the development of salt tolerance in crops. Plant Growth Regul. 2019, 89, 1-17. [CrossRef]

9. Yang, A.; Dai, X.; Zhang, W.H. A R2R3-type MYB gene, OsMYB2, is involved in salt, cold, and dehydration tolerance in rice. J. Exp. Bot. 2012, 63, 2541-2556. [CrossRef]

10. Hu, H.; You, J.; Fang, Y.; Zhu, X.; Qi, Z.; Xiong, L. Characterization of transcription factor gene SNAC2 conferring cold and salt tolerance in rice. Plant Mol. Biol. 2008, 67, 169-181. [CrossRef] [PubMed]

11. Wang, C.; Lu, G.; Hao, Y.; Guo, H.; Guo, Y.; Zhao, J.; Cheng, H. ABP9, a maize bZIP transcription factor, enhances tolerance to salt and drought in transgenic cotton. Planta 2017, 246, 453-469. [CrossRef]

12. Liang, Y.; Li, X.; Zhang, D.; Gao, B.; Yang, H.; Wang, Y.; Guan, K.; Wood, A.J. ScDREB8, a novel A-5 type of DREB gene in the desert moss Syntrichia caninervis, confers salt tolerance to Arabidopsis. Plant Physiol. Biochem. 2017, 120, 242-251. [CrossRef]

13. Bo, C.; Chen, H.; Luo, G.; Li, W.; Zhang, X.; Ma, Q.; Cheng, B.; Cai, R. Maize WRKY114 gene negatively regulates salt-stress tolerance in transgenic rice. Plant Cell Rep. 2020, 39, 135-148. [CrossRef]

14. Hao, Y.J.; Wei, W.; Song, Q.X.; Chen, H.W.; Zhang, Y.Q.; Wang, F.; Zou, H.F.; Lei, G.; Tian, A.G.; Zhang, W.K.; et al. Soybean NAC transcription factors promote abiotic stress tolerance and lateral root formation in transgenic plants. Plant J. 2011, 68, 302-313. [CrossRef]

15. He, Y.; Li, W.; Lv, J.; Jia, Y.; Wang, M.; Xia, G. Ectopic expression of a wheat MYB transcription factor gene, TaMYB73, improves salinity stress tolerance in Arabidopsis thaliana. J. Exp. Bot. 2012, 63, 1511-1522. [CrossRef] [PubMed]

16. Peng, X.J.; Xingyong, M.; Weihong, F.; Man, S.; Liqin, C.; Alam, I.; Lee, B.H.; Dongmei, Q.; Shihua, S.; Gongshe, L. Improved drought and salt tolerance of Arabidopsis thaliana by transgenic expression of a novel DREB gene from Leymus chinensis. Plant Cell Rep. 2011, 30, 1493-1502.

17. Zhao, P.; Li, X.; Jia, J.; Yuan, G.; Chen, S.; Qi, D.; Cheng, L.; Liu, G. LcbHLH92 from sheepgrass acts as a negative regulator of anthocyanin/proanthocyandin accumulation and influences seed dormancy. J. Exp. Bot. 2019, 70, 269-284. [CrossRef] [PubMed]

18. Szabados, L.; Savoure, A. Proline: A multifunctional amino acid. Trends Plant Sci. 2010, 15, 89-97. [CrossRef] [PubMed]

19. Zheng, J.; Su, H.; Lin, R.; Zhang, H.; Xia, K.; Jian, S.; Zhang, M. Isolation and characterization of an atypical LEA gene (IpLEA) from Ipomoea pes-caprae conferring salt/drought and oxidative stress tolerance. Sci. Rep. 2019, 9, 14838. [CrossRef]

20. Xu, Y.; Yu, Z.; Zhang, S.; Wu, C.; Yang, G.; Yan, K.; Zheng, C.; Huang, J. CYSTM3 negatively regulates salt stress tolerance in Arabidopsis. Plant Mol. Biol. 2019, 99, 395-406. [CrossRef]

21. Lu, P.; Magwanga, R.O.; Kirungu, J.N.; Hu, Y.; Dong, Q.; Cai, X.; Zhou, Z.; Wang, X.; Zhang, Z.; Hou, Y.; et al. Overexpression of Cotton a DTX/MATE Gene Enhances Drought, Salt, and Cold Stress Tolerance in Transgenic Arabidopsis. Front Plant Sci. 2019, 10, 299. [CrossRef]

22. Nevo, E.; Chen, G. Drought and salt tolerances in wild relatives for wheat and barley improvement. Plant Cell Environ. 2010, 33, 670-685. [CrossRef]

23. Chen, S.; Huang, X.; Yan, X.; Liang, Y.; Wang, Y.; Li, X.; Peng, X.; Ma, X.; Zhang, L.; Cai, Y.; et al. Transcriptome analysis in sheepgrass (Leymus chinensis): A dominant perennial grass of the Eurasian Steppe. PLoS ONE 2013, 8, e67974. [CrossRef]

24. Gao, Q.; Li, X.; Jia, J.; Zhao, P.; Liu, P.; Liu, Z.; Ge, L.; Chen, S.; Qi, D.; Deng, B.; et al. Overexpression of a novel cold-responsive transcript factor LcFIN1 from sheepgrass enhances tolerance to low temperature stress in transgenic plants. Plant Biotechnol. J. 2016, 14, 861-874. [CrossRef] [PubMed]

25. Cheng, L.; Li, X.; Huang, X.; Ma, T.; Liang, Y.; Ma, X.; Peng, X.; Jia, J.; Chen, S.; Chen, Y.; et al. Overexpression of sheepgrass R1-MYB transcription factor LcMYB1 confers salt tolerance in transgenic Arabidopsis. Plant Physiol. Biochem. 2013, 70, 252-260. [CrossRef]

26. Ma, T.; Li, M.; Zhao, A.; Xu, X.; Liu, G.; Cheng, L. LcWRKY5: An unknown function gene from sheepgrass improves drought tolerance in transgenic Arabidopsis. Plant Cell Rep. 2014, 33, 1507-1518. [CrossRef] [PubMed]

27. Liu, Z.; Yuan, G.; Liu, S.; Jia, J.; Cheng, L.; Qi, D.; Shen, S.; Peng, X.; Liu, G. Identified of a novel cis-element regulating the alternative splicing of LcDREB2. Sci. Rep. 2017, 7, 46106. [CrossRef]

28. Li, X.; Hou, S.; Gao, Q.; Zhao, P.; Chen, S.; Qi, D.; Lee, B.H.; Cheng, L.; Liu, G. LcSAIN1, a novel salt-induced gene from sheepgrass, confers salt stress tolerance in transgenic Arabidopsis and rice. Plant Cell Physiol. 2013, 54, 1172-1185. [CrossRef] 
29. Li, X.; Gao, Q.; Liang, Y.; Ma, T.; Cheng, L.; Qi, D.; Liu, H.; Xu, X.; Chen, S.; Liu, G. A novel salt-induced gene from sheepgrass, LcSAIN2, enhances salt tolerance in transgenic Arabidopsis. Plant Physiol. Biochem. 2013, 64, 52-59. [CrossRef]

30. Li, X.; Yang, W.; Liu, S.; Li, X.Q.; Jia, J.; Zhao, P.; Cheng, L.; Qi, D.; Chen, S.; Liu, G. LcFIN2, a novel chloroplast protein gene from sheepgrass, enhances tolerance to low temperature in Arabidopsis and rice. Physiol. Plant. 2019, 166, 628-645. [CrossRef]

31. Chou, K.C.; Shen, H.B. Plant-mPLoc: A top-down strategy to augment the power for predicting plant protein subcellular localization. PLoS ONE 2010, 5, e11335. [CrossRef]

32. Livak, K.J.; Schmittgen, T.D. Analysis of relative gene expression data using real-time quantitative PCR and the 2(T)(-Delta Delta C) method. Methods 2001, 25, 402-408. [CrossRef]

33. Clough, S.J.; Bent, A.F. Floral dip: A simplified method for Agrobacterium-mediated transformation of Arabidopsis thaliana. Plant J. 1998, 16, 735-743. [CrossRef]

34. Zhao, Y.; Yang, Z.; Ding, Y.; Liu, L.; Han, X.; Zhan, J.; Wei, X.; Diao, Y.; Qin, W.; Wang, P.; et al. Over-expression of an R2R3 MYB Gene, GhMYB73, increases tolerance to salt stress in transgenic Arabidopsis. Plant Sci. 2019, 286, 28-36. [CrossRef] [PubMed]

35. Shan, D.P.; Huang, J.G.; Yang, Y.T.; Guo, Y.H.; Wu, C.A.; Yang, G.D.; Gao, Z.; Zheng, C.C. Cotton GhDREB1 increases plant tolerance to low temperature and is negatively regulated by gibberellic acid. New Phytol. 2007, 176, 70-81. [CrossRef]

36. Durak, I.; Yurtarslanl, Z.; Canbolat, O.; Akyol, O. A methodological approach to superoxide dismutase (SOD) activity assay based on inhibition of nitroblue tetrazolium (NBT) reduction. Clin. Chim. Acta 1993, 214, 103-104. [CrossRef]

37. Wang, L.; Li, X.; Chen, S.; Liu, G. Enhanced drought tolerance in transgenic Leymus chinensis plants with constitutively expressed wheat TaLEA3. Biotechnol. Lett. 2009, 31, 313-319. [CrossRef]

38. Yokotani, N.; Higuchi, M.; Kondou, Y.; Ichikawa, T.; Iwabuchi, M.; Hirochika, H.; Matsui, M.; Oda, K. A novel chloroplast protein, CEST induces tolerance to multiple environmental stresses and reduces photooxidative damage in transgenic Arabidopsis. J. Exp. Bot. 2011, 62, 557-569. [CrossRef]

39. Wang, F.; Yang, C.L.; Wang, L.L.; Zhong, N.Q.; Wu, X.M.; Han, L.B.; Xia, G.X. Heterologous expression of a chloroplast outer envelope protein from Suaeda salsa confers oxidative stress tolerance and induces chloroplast aggregation in transgenic Arabidopsis plants. Plant Cell Environ. 2012, 35, 588-600. [CrossRef] [PubMed]

40. Nawaz, G.; Kang, H. Rice OsRH58, a chloroplast DEAD-box RNA helicase, improves salt or drought stress tolerance in Arabidopsis by affecting chloroplast translation. BMC Plant Biol. 2019, 19, 17. [CrossRef] [PubMed]

41. Zsigmond, L.; Szepesi, A.; Tari, I.; Rigo, G.; Kiraly, A.; Szabados, L. Overexpression of the mitochondrial PPR40 gene improves salt tolerance in Arabidopsis. Plant Sci. 2012, 182, 87-93. [CrossRef] [PubMed]

42. Xu, N.; Chu, Y.; Chen, H.; Li, X.; Wu, Q.; Jin, L.; Wang, G.; Huang, J. Rice transcription factor OsMADS25 modulates root growth and confers salinity tolerance via the ABA-mediated regulatory pathway and ROS scavenging. PLoS Genet. 2018, 14, e1007662. [CrossRef] [PubMed]

43. Msanne, J.; Lin, J.; Stone, J.M.; Awada, T. Characterization of abiotic stress-responsive Arabidopsis thaliana RD29A and RD29B genes and evaluation of transgenes. Planta 2011, 234, 97-107. [CrossRef] [PubMed]

44. Han, G.; Yuan, F.; Guo, J.; Zhang, Y.; Sui, N.; Wang, B. AtSIZ1 improves salt tolerance by maintaining ionic homeostasis and osmotic balance in Arabidopsis. Plant Sci. 2019, 285, 55-67. [CrossRef] [PubMed] 